\title{
Contrast mechanisms associated with Neuromelanin-MRI
}

Paula Trujillo ${ }^{1,2}$, Paul Summers ${ }^{1}$, Emanuele Ferrari ${ }^{3}$, Fabio A. Zucca ${ }^{3}$, Michela Sturini ${ }^{4}$, Luca Mainardi ${ }^{2}$, Sergio Cerutti $^{2}$, Alex K Smith ${ }^{5,6}$, Seth A Smith ${ }^{5,6,7}$, Luigi Zecca ${ }^{3}$, Antonella Costa ${ }^{1}$

${ }^{1}$ Department of Neuroradiology, Fondazione IRCCS Ca' Granda - Ospedale Maggiore Policlinico, Milan, MI, Italy

${ }^{2}$ Department of Electronics, Information and Bioengineering, Politecnico di Milano, Milan, MI, Italy

${ }^{3}$ Institute of Biomedical Technologies, National Research Council of Italy, Segrate, MI, Italy

${ }^{4}$ Department of Chemistry, University of Pavia, Pavia, PV, Italy

${ }^{5}$ Vanderbilt University Institute of Imaging Science, Vanderbilt University, Nashville, TN, USA

${ }^{6}$ Department of Biomedical Engineering, Vanderbilt University, Nashville, TN, USA

${ }^{7}$ Department of Radiology and Radiological Sciences, Vanderbilt University, Nashville, TN, USA

\section{Full postal and email address of the corresponding author:}

Paula Trujillo

Fondazione IRCCS Ca' Granda - Ospedale Maggiore Policlinico

Department of Neuroradiology

Via F. Sforza 35

20122, Milan, MI, Italy

Email: paula.trujillo@polimi.it

\begin{abstract}
Abbreviations: $\mathrm{BSA}=$ bovine serum albumin; DS = direct saturation; $\mathrm{LC}=$ locus coeruleus; $\mathrm{MT}=$ magnetization transfer; $\mathrm{NM}=$ Neuromelanin; $\mathrm{PD}=$ Parkinson's Disease; PSR = pool size ratio; $\mathrm{qMT}=$ quantitative MT; RF = radiofrequency; $\mathrm{SN}=$ Substantia Nigra; TSE = turbo spin echo.
\end{abstract}




\section{Abstract}

Purpose: To investigate the physical mechanisms associated with the contrast observed in neuromelanin-MRI.

Methods: Phantoms having different concentrations of synthetic melanins with different degrees of iron loading were examined on a 3T scanner using relaxometry and quantitative magnetization transfer (MT).

Results: Concentration-dependent $\mathrm{T}_{1^{-}}$and $\mathrm{T}_{2}$-shortening was most pronounced for the melanin pigment when combined with iron. Metal-free melanin had a negligible effect on the magnetization transfer spectra. On the contrary, the presence of iron-laden melanins resulted in a decreased magnetization transfer ratio. The presence of melanin or iron (or both) did not have a significant effect on the macromolecular content, represented by the pool size ratio.

Conclusion: The primary mechanism underlying contrast in neuromelanin-MRI seems to be the $\mathrm{T}_{1}$ reduction associated with melanin-iron complexes. The macromolecular content is not significantly influenced by the presence of melanin with or without iron, and thus the MT is not directly affected. However, as $\mathrm{T}_{1}$ plays a role in determining the MT-weighted signal, the magnetization transfer ratio is reduced in the presence of melanin-iron complexes.

Keywords: neuromelanin, MRI, magnetization transfer, relaxation 


\section{Introduction}

Neuromelanin (NM) is a dark complex pigment present in several types of neurons, and particularly concentrated in the dopaminergic neurons of the substantia nigra pars compacta $(\mathrm{SNc})$ and the noradrenergic neurons of the locus coeruleus (LC) $(1,2)$. Inside neurons, NM pigment is accumulated within cytoplasmic organelles surrounded by a double membrane, together with lipid bodies and proteins $(3,4)$. NM pigment itself is composed of different components: melanin, proteins, lipids and metal ions. Among the metals ions, iron is the most abundant and it has been demonstrated that NM is the main iron storage in pigmented neurons of the SNc $(5,6)$. NM accumulates normally with age (5), but is strongly depleted in patients with Parkinson's disease (PD)

(7) due to selective loss of NM-containing neurons (8-11), suggesting a role of NM in the neurodegenerative processes of PD.

Neuromelanin-MRI (NM-MRI) (12) has attracted attention as a method that may allow in-vivo assessment of NM and assist in disease characterization. NM-MRI is typically based on a $\mathrm{T}_{1}$-weighted turbo spin echo (TSE) pulse sequence, and has been found to provide notable contrast between both the SNc and LC and surrounding brain tissues (13-15). The first study describing the NM-MRI technique suggested paramagnetic $\mathrm{T}_{1}$-shortening associated with the presence of NM as an origin for the high signal intensity of the SNc (12). In addition to the iron associated with NM, the SNc also has a high concentration of iron in the form of ferritin (16), that has also been suggested as a cause of $\mathrm{T}_{1}$-shortening effects in NM-MRI (12). Notably however, other iron-laden structures in the brain, for example the red nucleus, do not exhibit particular hyperintensity in NM-MRI. Moreover, in a direct comparison between post-mortem NM-MRI and neuropathological findings, Kitao et al. found NM-MRI signal intensity in the SNc to be closely associated with the quantity of NM-containing neurons, whereas the increased signal intensity in the SNc did not seem to be influenced by iron deposition (17). Whilst the study by Kitao et al. provides evidence of a link between NM-MRI hyperintensity and the presence of NM, the mechanism by which the presence of NM might give rise to signal hyperintensities, and the nature of the relationship between a loss of hyperintensity with advancing PD and NM status of the tissues are still unclear.

In the initial work by Sasaki et al. (12), which was carried out at 3T, the authors attributed the NM-MRI contrast mainly to $\mathrm{T}_{1}$-shortening effects and they attributed a failure to depict NM-related contrast at lower field strengths (i.e. up to $1.5 \mathrm{~T}$ ) to the negligible $\mathrm{T}_{1}$ difference between neuromelanin-containing and surrounding brain tissue. Subsequent observations that magnetization transfer (MT) preparation of NM-MRI pulse sequences increases the contrast however, have led several authors to credit MT as the primary source of contrast (18-20). Indeed, NMMRI is typically based on a $\mathrm{T}_{1}$-weighted TSE sequences which are subject to incidental MT effects associated with the extended train of refocusing pulses in multi-slice acquisition (21). Moreover, we (22), and others $(15,18,19,23,24)$ have used explicit MT effects, generated by applying MT preparation pulses to 3D gradient echo pulse sequences (that have little intrinsic MT weighting), to establish contrast between NM-containing structures and their surroundings, even at 1.5T (18). However, the above-mentioned studies of NM contrast lack 
calibration against standards at known melanin content, and no characterization of the MT properties associated with NM has been performed.

MT contrast is the result of interaction and exchange between water protons and the protons associated with macromolecules (25). The MT effect has often been characterized via the MT ratio (MTR) (26), and more recently via quantitative MT (qMT), which involves acquiring images at multiple RF offsets to generate the MTR-spectrum (27). The resulting MTR-spectrum can be fitted to a model (28-32) to estimate quantitative indices, such as the macromolecular-to-free pool size ratio $(P S R)$, the MT exchange rates $\left(k_{f m}, k_{m f}\right)$, and the

longitudinal and transverse relaxation times for each pool $\left(T_{1}^{f, m}, T_{2}^{f, m}\right)$. At low offset frequencies, the RF pulse will inadvertently and partially saturate the free pool via direct saturation, (DS) and attenuate the observed signal (33). Consequently, the observed signal attenuation is due to both MT and DS effects.

While it has been suggested that MT plays a role on NM-MRI contrast, there are no reports providing quantitative evidence of a preferential MT effect in the presence of NM. The paramagnetic effect of NM increases when combined with metals such as iron and copper, and prior in vitro experiments have revealed concentration-dependent $\mathrm{T}_{1}$-shortening effects of synthetic melanins (34-36). These observations however, do not explain the enhancement of contrast between NM-containing structures and surrounding tissues seen when MT preparation is performed. The MT effect is known to enhance paramagnetic contrast $(37,38)$, and sequences with an explicit MT contrast preparation pulse have shown higher sensitivity in detecting NM than those relying on incidental MT effects $(19,20)$.

We investigated the roles played by NM and iron in determining relaxations times and pool size ratio by examining the MTR-spectra of phantoms containing iron and complexes of synthetic melanin-iron at different concentrations similar to those observed in human SNc tissue. We also performed simulations of the Bloch equations of the two-pool system to assess the effect of pool parameters on the MTR-spectra.

\section{Methods}

\section{Theory and Simulation}

In the presence of MT, the time evolutions of the free and macromolecular pools can be described by the Bloch equations for two spin populations coupled by exchange (39):

$$
\frac{d \mathbf{M}(t)}{d t}=\mathbf{A}(t) \mathbf{M}(t)+\mathbf{B}
$$

where $\mathbf{M}$ is the magnetization vector with the $\mathrm{x}, \mathrm{y}$, and z-components of magnetization of the free $\left({ }^{f}\right)$ and macromolecular $\left(^{\mathrm{m}}\right)$ pools $\left(M_{x}^{f}, M_{y}^{f}, M_{z}^{f}, M_{z}^{m}\right)$, and $\mathbf{A}$ and $\mathbf{B}$ are matrices corresponding to the coefficients of the Bloch equations: 


$$
\begin{gathered}
\mathbf{A}=\left[\begin{array}{cccc}
-R_{2}^{f} & -\Delta \omega & 0 & 0 \\
\Delta \omega & -R_{2}^{f} & -\omega_{1}(t) & 0 \\
0 & \omega_{1}(t) & -\left(R_{1}^{f}+k_{f m}\right) & k_{m f} \\
0 & 0 & k_{f m} & -\left(R_{1}^{m}+k_{m f}+W(t)\right)
\end{array}\right] \\
\mathbf{B}=\left[\begin{array}{c}
0 \\
0 \\
R_{1}^{f} M_{0}^{f} \\
R_{1}^{m} M_{0}^{m}
\end{array}\right]
\end{gathered}
$$

where $R_{1,2}^{f, m}$ are the longitudinal and transverse relaxation rate of each pool $\left(R_{1,2}^{f, m}=1 / T_{1,2}^{f, m}\right), \Delta \omega$ is the frequency offset of the RF pulse, $\omega_{1}(t)$ is time-varying amplitude of the applied RF pulse, $M_{0}^{f, m}$ are the equilibrium magnetization of each pool, $k_{m f}$ is the rate of MT exchange from the macromolecular pool to the free pool, and $k_{f m}$ is exchange rate in the other direction $\left(k_{f m}=k_{m f} * M_{0}^{m} / M_{0}^{f}\right)$. The term $W(t)$ is the saturation rate of the macromolecular pool and is defined as $W(t)=\pi \omega_{1}^{2}(t) g\left(\Delta \omega, T_{2}^{m}\right)$, where $g\left(\Delta \omega, T_{2}^{m}\right)$ is the absorption line shape for the macromolecular pool, typically chosen to be a super-Lorentzian or Gaussian (40).

The magnitude of the MT effect depends on the frequency offset $(\Delta \omega)$, power, and duration of the RF pulse, and can be semi-quantitatively represented by the MTR-spectrum:

$$
\operatorname{MTR}(\Delta \omega)=1-\frac{S(\Delta \omega)}{S_{0}}
$$

where $S(\Delta \omega)$ and $S_{0}$ are the voxel intensities in the presence and absence of RF irradiation, respectively. In the absence of $\mathrm{MT}\left(k_{m f}=0\right)$, the Bloch equations are simplified, and represent the DS of water protons.

We performed a series of simulations in MATLAB (R2015a, Mathworks, Natick, MA) using the Bloch equations to assess the effect of spin system parameters on the MTR spectrum. We simulated the MT effect for different spin system parameters $\left(T_{1}^{f}=0.25,0.5,0.75,1 \mathrm{~s} ; T_{2}^{f}=30,60,120,240 \mathrm{~ms} ; T_{2}^{m}=8,14,20,26 \mu \mathrm{s}\right.$; $M_{0}^{m}=0.005,0.01,0.03,0.06 ; k_{f m}=0.1,0.5,1,10 \mathrm{~s}^{-1}$ ) and different pulse amplitudes (effective saturation flip angle, $\alpha_{\mathrm{MT}}=400^{\circ}, 650^{\circ}, 900^{\circ}, 1150^{\circ}$ ). In all cases, the values of the remaining parameters were fixed at the following values: $T_{1}^{f}=1 \mathrm{~s}, T_{1}^{m}=1 \mathrm{~s}, T_{2}^{f}=240 \mathrm{~ms}, T_{2}^{m}=14 \mu \mathrm{s}, M_{0}^{f}=1, M_{0}^{m}=0.01, k_{f m}=1 \mathrm{~s}^{-1}$. The $T_{1}^{f}$ and $T_{2}^{f}$ values, and the choice of a super-Lorentzian absorption lineshape for the macromolecular pool were based on preliminary testing with polyacrylamide gels. The RF pulse modeled in this series of simulations utilized the same parameters as the subsequent MT experimental protocol (25-ms sinc-gauss, $\alpha_{\mathrm{MT}}=1150^{\circ}$, excitation flip angle $\left.=10^{\circ}, \mathrm{TR}=58 \mathrm{~ms}\right)$. In accordance with previous studies $(29,31,41)$, we assumed that the sequence was ideally spoiled, and the analytical solution was repeated for consecutive time intervals of the pulse sequence until the pulsed steady state was achieved. The MTR-spectrum was obtained by applying the RF pulse at different offset frequencies logarithmically spaced from 0.1 to $100 \mathrm{kHz}$, and the obtained $M_{z}^{f}$ values were 
normalized to the value computed using $\alpha_{\mathrm{MT}}=0$. In order to examine the DS effects, we also solved the Bloch equations in the absence of MT $\left(k_{f m}=0\right)$ using the same $T_{1}^{f}, T_{2}^{f}$, and RF pulse as above.

\section{Experimental}

\section{Preparation of synthetic melanins}

The melanin was synthesized by autoxidation of dopamine in presence of L-Cysteine as previously described (1) with minor changes to obtain pheomelanin. Metallomelanins containing different iron loadings were prepared by dissolving dopamine $(225 \mathrm{mg})$, L-Cysteine $\left(29.7 \mathrm{mg}\right.$ ) and different amounts of $\mathrm{Fe}\left(\mathrm{NH}_{4}\right)_{2}\left(\mathrm{SO}_{4}\right)_{2} \bullet 6 \mathrm{H}_{2} \mathrm{O}$ in $100 \mathrm{ml}$ of $50 \mathrm{mM}$ phosphate buffer ( $\mathrm{pH}$ 7.4) prepared with purified water (Milli-Q®). The mixture was allowed to react in air, at $37{ }^{\circ} \mathrm{C}$, protected from light for 4 days. The resulting suspension of synthetic melanin pigment was then centrifuged at $8000 \mathrm{rpm}$ for $20 \mathrm{~min}$ in order to remove unreacted dopamine and small soluble oligomers of dopamine. The pellet was then washed with an acetic acid solution (1\% v/v) and a $\mathrm{NaCl}$ solution (9\% w/v). The product was incubated with $25 \mathrm{ml}$ of a $0.15 \mathrm{M}$ solution of Na2-EDTA for $8 \mathrm{~h}$, centrifuged and suspended again with $25 \mathrm{ml}$ of Na2-EDTA solution $0.15 \mathrm{M}$ for $14 \mathrm{~h}$, in order to remove unbound iron ions. After several washes with purified water, the melanin was freeze-dried and stored at $-20{ }^{\circ} \mathrm{C}$. Table 1 reports the synthesis conditions of the prepared melanins. Analysis of the iron content was performed by means of ICP-OES after treatment with purified water $65 \% \mathrm{HNO}_{3}(42)$. The amounts of iron bound to the synthetic melanins were similar to those found in NM isolated from SNc of healthy subjects (4-6). Melanins were suspended in purified water to obtain a homogenous suspension with a concentration of $14 \mathrm{mg} / \mathrm{ml}$.

\section{Preparation of phantoms}

As relaxation rates play a role in determining the MT effect (Eq. 1), we performed a series of preliminary measurements in different gels to arrive at a relatively close approximation of in-vivo relaxation properties subject to the constraints imposed by working with melanins (see Supplementary Material). The polyacrylamide gel used for the experiments herein consisted of 10\% (w/v) BSA (Sigma-Aldrich A4503) in 10\% Acrylamide/Bis-Acrylamide mixture (37.5:1) in $0.4 \mathrm{M}$ Tris- $\mathrm{HCl}, \mathrm{pH} 7.4$, prepared using the protocol for polyacrylamide gels electrophoresis (43), but without using sodium dodecyl sulfate. The appropriate quantities of the melanin preparations were then suspended in the base gel. Care was taken to suspend the melanins as rapidly and uniformly as possible while avoiding air bubble formation and sedimentation.

In addition to the samples containing synthetic melanins, we also prepared four samples containing only iron. The iron present was a $\mathrm{Fe}$ (III)-EDTA complex obtained by dissolving $\mathrm{Fe}_{2}\left(\mathrm{SO}_{4}\right)_{3}$ and $\mathrm{Na}_{2}$-EDTA with a $\mathrm{Fe}(\mathrm{III}) / \mathrm{EDTA}$ molar ratio 1:3. Table 2 summarizes the compositions of the prepared samples. 
Having observed that the MT spectra of the polyacrylamide gels was notably different from those seen in vivo and in prior reports for phantoms $(25,44)$, we prepared a second phantom using $4 \%$ agarose (low gelling temperature agarose, Sigma-Aldrich) as the base gel, which previous measurements had shown to have a significant MT effect. Table 3 summarizes the compositions of the second phantom.

\section{MRI Acquisition}

All MR images were obtained on a clinical 3T scanner (Achieva, Philips Medical Systems, Best, the Netherlands) with a 32-channel head coil. Prior to imaging, the phantom was allowed to reach room temperature. The phantom was positioned with the tubes parallel to the magnet bore and subjected to qMT and DS measurements and an NM-MRI scan, as well as $\mathrm{T}_{1}, \mathrm{~T}_{2}, \mathrm{~B}_{0}$ and $\mathrm{B}_{1}$ mapping. All scans were executed in the axial plane with a 220x220x12 $\mathrm{mm}^{3}$ field of view, with the remaining scan details as described below.

For the qMT measurement, images were acquired using a 3D MT-prepared SPGR sequence (41) with a multishot EPI readout, TR $/ \mathrm{TE} / \alpha=58 \mathrm{~ms} / 7.9 \mathrm{~ms} / 10^{\circ}$, SENSE factor $=2$, EPI factor $=5$, resolution $=1 \times 1 \mathrm{~mm}^{2}$ inplane, slice thickness $=3 \mathrm{~mm}, \mathrm{NSA}=4$. MT preparation was achieved using a $25 \mathrm{~ms}$, single-lobed sinc-gauss pulse, $\alpha_{\mathrm{MT}}=400^{\circ}, 650^{\circ}, 900^{\circ}$ and $1150^{\circ}$, and 16 offset frequencies logarithmically spaced from 0.1 to $100 \mathrm{kHz}$. The $10^{\circ}$ excitation pulse used for image generation was kept fixed across all the MT preparations. Direct saturation (DS) data were also acquired using a 3D MT-prepared SPGR sequence (see Supporting Material).

$\mathrm{B}_{1}$ and $\mathrm{B}_{0}$ maps were acquired using $3 \mathrm{D}$ gradient echo sequences. $\mathrm{B}_{0}$ maps were obtained using the dual-TE phase-difference method (45) with $\mathrm{TR} / \mathrm{TE}_{1} / \mathrm{TE}_{2}=50 / 5.8 / 8.1 \mathrm{~ms}$ and $\alpha=25^{\circ} . \mathrm{B}_{1}$ maps were obtained using the actual flip-angle imaging method (46) with $\mathrm{TR}_{1} / \mathrm{TR}_{2} / \mathrm{TE}=30 / 130 / 5.7 \mathrm{~ms}$ and $\alpha=60^{\circ}$.

$\mathrm{T}_{1}$ mapping was performed using a 3D multiple flip angle acquisition with $\mathrm{TR} / \mathrm{TE}=20 / 6.3 \mathrm{~ms}$ and $\alpha=5,10,15$, 20, 25, 30 ${ }^{\circ}$. $\mathrm{T}_{1}$ maps were reconstructed by fitting the Ernst equation with $\mathrm{B}_{1}$ correction (47). $\mathrm{T}_{2}$ mapping was performed using a 2D multi-slice multi-echo TSE acquisition with TR $/ \alpha=2000 \mathrm{~ms} / 90^{\circ}, \mathrm{TE}=40,60,80,100$, $120 \mathrm{~ms}$, from which quantitative $\mathrm{T}_{2}$ maps were calculated using the scanner manufacturer-supplied software.

Finally, we acquired a NM-MRI scan consisting of a $\mathrm{T}_{1}$-weighted TSE sequence with off-resonance magnetization transfer preparation pulses $(\mathrm{TE} / \mathrm{TR}=12 / 670 \mathrm{~ms}$, echo train length $=6$, resolution $=1 \times 1 \times 3.0 \mathrm{~mm}$, $\mathrm{NSA}=4)$.

Acquisition times were 27 min for qMT acquisition, 1 min $20 \mathrm{~s}$ for the $\mathrm{B}_{0}$ map, 1 min for the $\mathrm{B}_{1}$ map, $37 \mathrm{~s}$ for the $\mathrm{T}_{1}$ map, 3 min $48 \mathrm{~s}$ for the $\mathrm{T}_{2}$ map, and 4 min for the NM-MRI scan. 


\section{Image Analysis}

Image analysis was performed using MATLAB (R2015a, Mathworks, Natick, MA). Circular region of interest (ROI) of $10 \mathrm{~mm}$ diameter (roughly 77 voxels) were defined for each sample on the central slice. The average $\mathrm{T}_{1}$ $\left(T_{1}^{o b s}\right)$ and $\mathrm{T}_{2}\left(T_{2}^{o b s}\right)$ were measured for each ROI on the $\mathrm{T}_{1}$ and $\mathrm{T}_{2}$ maps, respectively. The relaxation rates $R_{1}^{o b s}$ and $R_{2}^{o b s}$ were calculated as the reciprocals of $T_{1}^{o b s}$ and $T_{2}^{o b s}$, respectively.

The qMT parameters were estimated on a voxel by voxel basis through multiparametric fitting to the two-pool MT model described in (32) modified as follows. The two-pool MT model contains six independent parameters: $R_{1}^{f}, R_{1}^{m}, T_{2}^{f}, T_{2}^{m}, P S R=M_{0}^{m} / M_{0}^{f}$, and $k_{f m}$. In keeping with conventional practice $(25,30,32,44)$, we assume $R_{1}^{m}$ to be $1 \mathrm{~s}^{-1}$ for all samples. $R_{1}^{o b s}\left(1 / T_{1}^{o b s}\right)$ values obtained from $\mathrm{T}_{1}$ mapping were used to determine the parameter $R_{1}^{f}$, as described by (25). In simulations (see results), we observed that varying $k_{f m}$ in the range $0.5-10 \mathrm{~s}^{-1}$ had little effect on the spectra, and we therefore used a fixed value $\left(k_{f m}=1 \mathrm{~s}^{-1}\right)$ for the fitting. The inverse exchange rate $k_{f m}$, corresponding to the ratio between $k_{f m}$ and $P S R$, thus does not represent an independent parameter. The three remaining parameters $\left(T_{2}^{f}, T_{2}^{m}\right.$, and $\left.P S R\right)$ were estimated by fitting the qMT data to the model. Before fitting, the signal intensities of the MT-weighted images were normalized to the intensity of the reference image without saturation, and the nominal offset frequency and RF amplitudes corrected using $\mathrm{B}_{0}$ and $\mathrm{B}_{1}$ maps, respectively. Only the points with frequency offset above $1 \mathrm{kHz}$ were considered for the fitting to avoid limitations in the treatment of DS effects within the MT model. Based on preliminary data, the absorption lineshape for the macromolecular pool was chosen to be a super-Lorentzian for the polyacrylamide phantom, and a Gaussian for the agarose phantom.

A representative point measurement of the MTR-spectrum was calculated at $\Delta \omega=1.5 \mathrm{kHz}$ (a frequency offset at which the DS effect is negligible and the MT effect is present), according to Eq. 4, for the MT data acquired with $\alpha_{\mathrm{MT}}=1150^{\circ}$. For this purpose, the experimental MTR-spectrum was interpolated to 500 data points to artificially increase the spectral resolution, the offset frequency was corrected using the $\mathrm{B}_{0}$ map, and the MTR value at 1.5 $\mathrm{kHz}$ was determined from the interpolated and offset-corrected data.

Finally, we used the NM-MRI images to calculate the contrast ratio (CNR) between each sample and the base gels as:

$$
C N R=\frac{I_{i}-I_{\text {ref }}}{I_{\text {ref }}}
$$

where $I_{i}$ corresponds to the mean signal intensity for sample $i$, and $I_{r e f}$ is the mean signal intensity for the base gel. 


\section{Statistical Analysis}

Statistical analyses were performed with JMP statistical package (version 11.2, SAS Institute, Inc., Cary, NC, USA). A multiple regression analysis was performed to investigate statistical dependencies amongst the melanin and iron concentrations and the MRI measures. The Spearman correlation coefficient was used to examine the correlation between measures.

\section{Results}

\section{Simulations}

Simulation results are shown in Figure 1. Decreasing $T_{1}^{f}$ reduced the MTR (Figure 1a), which can be attributed to narrowing the DS linewidth (Figure 1b), and decreasing the MT contributions at higher frequencies. On the contrary, reducing $T_{2}^{f}$ increased the DS linewidth and increased the MT contribution at lower frequencies for an overall increase in MTR (Figure 1c and Figure 1d). The effect of DS was negligible at frequencies higher than 2$3 \mathrm{kHz}$, with this upper limit being dependent on $\mathrm{T}_{1}$ and $\mathrm{T}_{2}$ of the free pool. Neither $T_{1}^{f}$ or $T_{2}^{f}$ altered MTR for frequencies higher than $10 \mathrm{kHz}$.

$T_{2}^{m}$ modulated the shape of the MTR-spectrum (Figure 1e). The macromolecular content altered the shape of the MTR-spectrum (Figure 1f), with a strong increase in MTR for increasing $M_{0}^{m}$. On the other hand, varying $k_{f m}$ in the range 0.5-10 s-1 resulted in a slight increase of the MTR (Figure 1g). We note however, that the impact of $k_{f m}$ scales with $M_{0}^{m}$, such that for a higher macromolecular content (e.g. $M_{0}^{m}=0.1$ ) the $k_{f m}$ had a more appreciable effect on the spectra, as reported by (31). Finally, we observed that increasing the MT pulse amplitude resulted in an augmented MT effect (Figure 1h).

\section{Experiments}

The metal-free melanin showed little effect on relaxation rates compared to the reference sample, while the metallomelanins showed an increase in both $R_{1}^{o b s}$ and $R_{2}^{o b s}$ with increasing metallomelanin concentration (Figure 2). The samples with only iron also showed increasing relaxation rates with increasing iron concentration, but for a given iron concentration, the metallomelanins produced even greater increases in $R_{1}^{o b s}$ and $R_{2}^{o b s}$ than iron alone (Figure 3). Expressed in terms of effective iron concentration, the relaxation rates of the metallomelanins showed greater relaxivities $\left(r_{1}=0.064 \mathrm{~s}^{-1} \mathrm{~mL} / \mu \mathrm{g}, \mathrm{r}_{2}=0.363 \mathrm{~s}^{-1} \mathrm{~mL} / \mu \mathrm{g}\right)$ than of iron alone $\left(\mathrm{r}_{1}=\right.$ $\left.0.024 \mathrm{~s}^{-1} \mathrm{~mL} / \mu \mathrm{g}, \mathrm{r}_{2}=0.035 \mathrm{~s}^{-1} \mathrm{~mL} / \mu \mathrm{g}\right)$. 
The shape of the MTR-spectrum for the unloaded polyacrylamide sample more closely resembled the simulations performed using a super-Lorentzian absorption lineshape for the macromolecular pool than a Gaussian lineshape, while, in agreement with previous studies (44), a Gaussian lineshape was appropriate for the $4 \%$ agarose gel.

All concentrations of the metal-free melanin showed similar spectra to the reference gel (Figure 4a). Increasing the iron loading for a given metallomelanin concentration (the maximum concentration being illustrated in Figure $4 \mathrm{~b}$ ), revealed two behaviors. With respect to the base gel, the samples with the lowest iron loading (metallomelanin 1) showed an increase in MTR at frequencies below $500 \mathrm{~Hz}$ and a reduction in MTR for frequencies between $500 \mathrm{~Hz}$ and $10 \mathrm{kHz}$. The MTRs of the metallomelanins with higher iron loadings were lower than those of the base gel above $300 \mathrm{~Hz}$, and of metallomelanin 1 at all frequencies below $10 \mathrm{kHz}$. Increasing the concentration of metallomelanin with a given iron loading (Figure 4c), resulted in a reduced MT effect though increasing the concentration above $1.75 \mathrm{mg} / \mathrm{ml}$ had minimal further effect on the spectra.

For the samples prepared in $4 \%$ agarose (Figure 5a) the shape of the MTR-spectra was similar to those previously reported for agar phantoms (44). For offset frequencies above $1 \mathrm{kHz}$, the experimental MTR-spectra corresponded closely to simulations based on the parameters as the qMT protocol, fixed $T_{2}^{m}=14 \mu \mathrm{s}, k_{f m}=2 \mathrm{~s}^{-}$ ${ }^{1}, M_{0}^{f}=1, M_{0}^{m}=0.015$, a Gaussian absorption lineshape for the macromolecular pool, and the measured $T_{1}^{o b s}$ and $T_{2}^{o b s}$ of the agarose samples (Figure 5b). The shape of the MTR-spectra of the agarose phantom differed from the spectra of the samples prepared in polyacrylamide. In particular, we found different behaviors of the spectra at low frequencies, possibly due to the differences in $T_{1}$ and $T_{2}$ in a range of frequencies dominated by DS effects. The decrease in MTR for the metallomelanins in agarose was observed across all measured offsets, whereas MTR measured in those prepared in polyacrylamide only showed a reduction for frequencies between $500 \mathrm{~Hz}$ and $10 \mathrm{kHz}$. Nevertheless, the effects of synthetic melanins at frequencies above $1 \mathrm{kHz}$ were comparable for both types of phantoms, with a negligible effect for the metal-free melanin and a decrease in MTR for the metallomelanins.

The values obtained from the qMT fitting are shown in Table 2 (polyacrylamide samples) and Table 3 (agarose samples). The estimated PSR values for the polyacrylamide samples (0.52-1.49\%; Table 2) were slightly lower than those for agarose samples $(0.90-1.69 \%$; Table 3$)$. The PSR was not significantly affected by the presence of metal-free melanin, iron, or metallomelanin in either case. In multiple regression analyses, the concentration of melanin and iron only explain $15-16 \%$ of the variability in the PSR values $\left(R^{2}=0.16, P=0.1516\right.$, root mean square error $(\mathrm{RMSE})=0.2172$ for the polyacrylamide phantom, and $\mathrm{R}^{2}=0.14, \mathrm{P}=0.4626, \mathrm{RMSE}=0.3211$ for the agarose phantom).

The estimated $T_{2}^{f}$ values were shorter than the $T_{2}^{o b s}$ values measured using the multiecho $\mathrm{T}_{2}$ mapping, but there was a significant correlation between the two measurements $(\rho=0.85, P<0.0001$ for the polyacrylamide samples, and $\rho=0.84, \mathrm{P}<0.001$ for the agarose samples). The $T_{2}^{m}$ values obtained for the reference and metal- 
free melanin prepared in polyacrylamide were higher than prior reports on both polyacrylamide and agar phantoms $(25,44,48)$, possibly because the absorption lineshape of the macromolecular pool is not wellrepresented by the Super-Lorentzian shape used for the fitting. We noticed, however, that the $T_{2}^{m}$ decreased with increasing concentrations of metallomelanins. The $T_{2}^{m}$ values for the samples in $4 \%$ agarose were close to the values previously reported for $4 \%$ agar $(14.36 \pm 2.48 \mu \mathrm{s})(44)$, and again diminished with increasing concentrations of metallomelanins.

The MTR calculated for $\alpha_{\mathrm{MT}}=1150^{\circ}$, and $\Delta \omega=1.5 \mathrm{kHz}$, decreased with increasing concentration of the metallomelanins (Figure 6a). Differently, the CNR calculated using the NM-MRI (Figure 6b) was increased for all the metallomelanins, but the signal intensity for the metallomelanins at the highest concentration was saturated, probably associated to a $\mathrm{T}_{2}$ effect (35).

\section{Discussion}

In this study, we examined a series of phantoms with different concentrations of synthetic melanins and iron (similar or slightly higher than found in human $\mathrm{SNc}(5,7)$ ) to investigate the role of melanin in relaxation times and saturation exchange processes. NM-MRI has attracted considerable interest as a potential means of quantifying neuronal loss in the SNc of patients with PD $(12-15,17)$. The source of NM-contrast, however, is still unclear. Early studies attributed its primary source to $\mathrm{T}_{1}$ effects (12), while more recently MT effects have been credited with providing most of the contrast between NM containing structures and neighboring tissues $(18,23,24)$.

It has been suggested that the presence of iron affects the MTR-spectrum $(33,41)$, and hence a mechanism exists for iron laden tissues to have distinct contrast on MT-prepared scans, but that does not explain the specificity of NM-MRI contrast to structures containing NM and not to other iron-rich regions. In human SN, the main iron storage protein is $\mathrm{H}$ - and L-ferritin, while the amount of iron present as NM-Fe complex is $10-20 \%$ of the total iron concentration in SN tissue (6). However, in dopamine NM-containing neurons of SNc the main iron compound is the NM-Fe complex $(5,6)$. In the NM-Fe complex there is one pool of iron as high spin Fe(III) in a mononuclear, rhombic structure, coordinated by six oxygen atoms and bound to the melanic component of NM (49-51). The other iron pool is made of multinuclear complexes of iron ions connected by oxy-hydroxy bridges similar to that present in ferritins $(6,52)$. Thus, deviations of the MR relaxation and exchange behaviors of metallomelanins from those of ferritins can be expected to depend on the size and exposure of the melanic bound pool.

In simulations, we found that $\mathrm{T}_{1}$ and $\mathrm{T}_{2}$ reductions introduce oppositely directed effects on the DS linewidth in the absence of magnetization exchange, with shorter $T_{2}$ being associated with broadening of the DS curve. This was also seen after the incorporation of magnetization exchange, as was a reduction of MTR associated with $\mathrm{T}_{1}$ 
reduction at frequencies above $2 \mathrm{kHz}$, where DS was negligible. Insomuch as DS is limited, and MT-related effects act primarily on the longitudinal component of the magnetization at higher frequencies $\mathrm{T}_{2}$ reduction had, as expected, no effect in this offset range. Thus, at high offset frequencies a reduction in MTR due to $\mathrm{T}_{1}$ shortening would not be countered by $\mathrm{T}_{2}$ shortening effects. At lower frequencies $(<2 \mathrm{kHz})$, the competing effects of $T_{1}$ and $T_{2}$ shortening on DS may lead to increased ( $T_{2}$ shortening dominant), decreased ( $T_{1}$ shortening dominant) or unchanged ( $T_{1}$ and $T_{2}$ shortening effects balanced) MTR due to changes in DS linewidth.

In fitting the qMT data from phantoms with two different base gels, we found neither iron, nor melanin nor metallomelanin to influence significantly the PSR. Consequently, the observed changes in the MTR-spectrum with changes in melanin and iron content would appear to be related to relaxation time effects (53). In agreement with previous studies $(36,35,34)$, we found concentration-dependent $T_{1}$ - and $T_{2}$-shortening to be far more pronounced for the metallomelanins than for similar concentrations of iron or metal-free melanin. Enochs et al., (35) attributed the decreased $T_{1}$, effects to the diamagnetic aggregated melanin and paramagnetic metal ions bound to melanin. Tosk et al., (34) later suggested that the strong interaction of iron and melanin in relaxation time shortening might be attributed to the formation of a superparamagnetic complex of ferric iron and melanin. Previous reports have also shown that paramagnetic substances (e.g. gadolinium-based paramagnetic agents) shorten the relaxation times of both free protons and a pool of restricted macromolecule protons, and consequently, the MT effect in the samples with paramagnetic ions is reduced (37).

In-vivo NM-MRI contrast may be the product of a combination of MT and T1 effects similar to MT-based background suppression in MR angiography (54). In a previous in-vivo study on healthy subjects (22), we found that MT-weighted 3D SPGR images with offset frequencies between $1 \mathrm{kHz}$ and $8 \mathrm{kHz}$ increases the hyperintensity in the location of the SNc, similar to those seen in TSE based NM-MRI, supporting the hypothesis that the contrast in NM-MRI might be associated with MT effects. In-vivo, the white matter has a higher macromolecular content (i.e. higher PSR values) and thus a stronger sensitivity to off-resonance RF pulses that results in greater saturation due to MT preparation than occurs in the SNc. Conversely, grey matter areas outside the brainstem have low PSR values (similar to those of the SNc), yet they do not appear hyperintense in NM-MRI. The exceptional relaxivity of metallomelanins likely contributes to the shorter T1 of the SNc that reduces its saturation due to $\mathrm{T}_{1}$-weighting (present in both the TSE and 3D-SPGR sequences) relative to other grey matter structures. Moreover, the simulations in the present study suggest that a dominance of T1 over T2 effects in relation to MT effects would account for the frequency selectivity of the MT increase in contrast seen in (22) and provide a further contribution to the SNc - grey matter contrast in NM-MRI. Our findings with iron-laden metallomelanins suggest that NM contributes to the exceptional combination of PSR and T1 that characterizes the SNc and favors SNc hyperintensity in NM-MRI where MT preparation and T1weighting lead to saturation of both white matter, due to higher PSR, and other grey matter structures, due to longer T1 and possibly wider MT z-spectra, than the SNc. 
Furthermore, the potent effect of metallomellanins on relaxation times relative to iron alone may explain several observations in the literature. First, only the SNc appears as an area of high signal intensity, while the SN pars reticulata (SNr), known to be rich in iron (55), does not appear bright in NM-MRI (19,56). In fact, SNr contains a lower amount of NM compared to SNc. Second, other iron-rich structures, such as the red nucleus (57), do not present the NM-MRI hyperintensity, indicating that iron alone is not the source of the contrast. Third, in patients with PD, there is decreasing contrast of the SNc and LC. Relative to other grey matter structures, NM is particularly abundant in the SNc and LC (58), thus, we propose, these structures are more subject to $\mathrm{T}_{1}$ and $\mathrm{T}_{2}$ shortening effects of metallomelanins than the other iron-rich areas. In patients with PD, the decreasing NM content may similarly reduce the relaxation (and by association MT) effects and so lead to the reduced contrast of the SNc and LC.

One must be aware however, that other processes may complicate application of this interpretation of NM-MRI. In particular, PD is also characterized by an overall increase of iron content throughout the SN (59-62) and an increase in the amount of iron bound to NM in PD (63-65) both of which may counter the effect of reduced NM $\mathrm{T}_{1}$ signal. As well, very high iron concentrations may lead to saturation of the signal intensity in TSE NM-MRI due to $\mathrm{T}_{2}$ shortening $(12,35)$. Furthermore, in $\mathrm{PD}$, the loss of pigmented dopaminergic neurons is also associated to other pathological changes in the SN, including the presence of Lewy bodies, which could also affect the relaxation times, the PSR and the exchange rate between pools. Further longitudinal in-vivo multi-imaging studies of the SN, possibly involving subjects with PD, may provide further evidence of the role of NM in generating MRI contrast.

This study was subject to several limitations. Although we were able to produce $T_{1}$ and $T_{2}$ values for the unloaded polyacrylamide phantom that were a reasonable approximation of gray matter values, MT properties of the gels used in this work are rather different from MT properties of tissues (i.e. lower PSR, faster exchange) which results in smaller baseline MTR. Moreover, it is likely that the absorption lineshape of the macromolecular pool of our experimental data cannot be represented by neither super-Lorentzian or Gaussian lineshapes commonly used in the literature. It was not our purpose in this paper to elucidate the degrees of quantitative correspondence to experimental measurements for each of the lineshapes, but rather to provide evidence of the effect of melanins in the MT spectra. For the purposes of reflecting the general impact of metallomelanin complexes on MT, therefore we consider our observations likely to be representative and we did not pursue a search for lineshapes better adapted to the data. As well, we did not perform susceptibility-weighted imaging, but in light of our results we recognize that quantitative susceptibility techniques $(66,67)$ may provide complementary information about the intrinsic magnetic properties of tissues. Lastly, the synthetic melanins used in this study lacked the lipidic and proteic components of the human NM (2,68). Although this approximation of the molecular structure may have implications for the relaxation and MT exchange processes, for the purpose of this study the synthetic models of NM here employed are acceptable since they reflect the iron binding in NM, which occurs via its melanic component and not to peptide or lipid moieties (69). 
In conclusion, our results show that the presence of melanin (with or without iron) does not affect the PSR, and therefore it does not directly alter the MT effect. However, as $\mathrm{T}_{1}$ plays a role in determining the MT z-spectrum width such that in consequence of T1 shortening, the MTR can be reduced in the presence of melanin-iron complexes. These findings support a view of NM-MRI contrast as a combination of MT and T1 effects, and should provide insight for the further optimization of NM-MRI sequences, with the purpose to diagnose PD before the onset of symptoms and to monitor the disease progression. Further longitudinal in-vivo multi-imaging studies of the SN may provide additional evidence of the role of NM in generating MRI contrast.

\section{Acknowledgements}

PT has a doctoral studentship funded by Fondazione IRCCS Ca' Granda Ospedale Maggiore Policlinico di Milano. FAZ, EF, and LZ were supported by Italian Ministry of Education, University, and Research (MIUR) National Research Programme (PNR) - CNR Flagship “InterOmics” Project (PB.P05) and by PNR - CNR Aging program 2012-2014. LZ also thanks the support of the Grigioni Foundation for Parkinson's disease, Milan, Italy.

\section{References}

1. Zecca L, Shima T, Stroppolo A, Goj C, Battiston GA, Gerbasi R, Sarna T, Swartz HM. Interaction of neuromelanin and iron in substantia nigra and other areas of human brain. Neuroscience 1996;73:407-15.

2. Zucca FA, Basso E, Cupaioli FA, Ferrari E, Sulzer D, Casella L, Zecca L. Neuromelanin of the human substantia nigra: an update. Neurotox. Res. 2014;25:13-23.

3. Sulzer D, Mosharov E, Talloczy Z, Zucca F a, Simon JD, Zecca L. Neuronal pigmented autophagic vacuoles: lipofuscin, neuromelanin, and ceroid as macroautophagic responses during aging and disease. J. Neurochem. 2008;106:24-36.

4. Zecca L, Bellei C, Costi $\mathrm{P}$, et al. New melanic pigments in the human brain that accumulate in aging and block environmental toxic metals. Proc. Natl. Acad. Sci. U. S. A. 2008;105:17567-72.

5. Zecca L, Stroppolo A, Gatti A, et al. The role of iron and copper molecules in the neuronal vulnerability of locus coeruleus and substantia nigra during aging. Proc. Natl. Acad. Sci. U. S. A. 2004;101:9843-8.

6. Zecca L, Gallorini M, Schünemann V, Trautwein AX, Gerlach M, Riederer P, Vezzoni P, Tampellini D. Iron, neuromelanin and ferritin content in the substantia nigra of normal subjects at different ages: consequences for iron storage and neurodegenerative processes. J. Neurochem. 2001;76:1766-73.

7. Zecca L, Fariello R, Riederer P, Sulzer D, Gatti A, Tampellini D. The absolute concentration of nigral neuromelanin, assayed by a new sensitive method, increases throughout the life and is dramatically decreased in 
Parkinson's disease. FEBS Lett. 2002;510:216-20.

8. Fearnley J, Lees A. Ageing and Parkinson's disease: substantia nigra regional selectivity. Brain 1991;114:2283-301.

9. German DC, Manaye KF, White CL, Woodward DJ, McIntire DD, Smith WK, Kalaria RN, Mann DM. Disease-specific patterns of locus coeruleus cell loss. Ann. Neurol. 1992;32:667-76.

10. Hirsch E, Graybiel A, Agid Y. Melanized dopaminergic neurons are differentially susceptible to degeneration in Parkinson's disease. Nature 1988;334:345-8.

11. Mann DM, Yates PO. Possible role of neuromelanin in the pathogenesis of Parkinson's disease. Mech. Ageing Dev. 1983;21:193-203.

12. Sasaki M, Shibata E, Tohyama K, Takahashi J, Otsuka K, Tsuchiya K, Takahashi S, Ehara S, Terayama Y, Sakai A. Neuromelanin magnetic resonance imaging of locus ceruleus and substantia nigra in Parkinson's disease. Neuroreport 2006;17:1215-1218.

13. Kashihara K, Shinya T, Higaki F. Neuromelanin magnetic resonance imaging of nigral volume loss in patients with Parkinson's disease. J Clin Neurosci 2011;18:1093-6.

14. Matsuura K, Maeda M, Yata K, Ichiba Y, Yamaguchi T, Kanamaru K, Tomimoto H. Neuromelanin magnetic resonance imaging in Parkinson's disease and multiple system atrophy. Eur Neurol 2013;70:70-7.

15. Schwarz S, Rittman T, Gontu V, Morgan P, Bajaj N, Auer D. T1-weighted MRI shows stage-dependent substantia nigra signal loss in Parkinson's disease. Mov. Disord. 2011;26:335-335.

16. Zecca L, Youdim MBH, Riederer P, Connor JR, Crichton RR. Iron, brain ageing and neurodegenerative disorders. Nat. Rev. Neurosci. 2004;5:863-73.

17. Kitao S, Matsusue E, Fujii S, Miyoshi F, Kaminou T, Kato S, Ito H, Ogawa T. Correlation between pathology and neuromelanin MR imaging in Parkinson's disease and dementia with Lewy bodies. Neuroradiology 2013;55:947-53.

18. Nakane T, Nihashi T, Kawai H, Naganawa S. Visualization of neuromelanin in the Substantia nigra and locus ceruleus at $1.5 \mathrm{~T}$ using a 3D-gradient echo sequence with magnetization transfer contrast. Magn. Reson. Med. Sci. 2008;7:205-10.

19. Langley J, Huddleston DE, Chen X, Sedlacik J, Zachariah N, Hu X. A multicontrast approach for comprehensive imaging of substantia nigra. Neuroimage 2015;112:7-13.

20. Schwarz ST, Bajaj N, Morgan PS, Reid S, Gowland P, Auer DP. Magnetisation Transfer contrast to enhance detection of neuromelanin loss at $3 \mathrm{~T}$ in Parkinson's disease. In: Proc. Intl. Soc. Mag. Reson. Med. Vol. 21. ; 2013. p. 2848. 
21. Dixon WT, Engels H, Castillo M, Sardashti M. Incidental magnetization transfer contrast in standard multislice imaging. Magn. Reson. Imaging 1990;8:417-22.

22. Trujillo P, Smith AK, Summers PE, Mainardi LM, Cerutti S, Smith SA, Costa A. High-resolution quantitative imaging of the substantia nigra. Conf. Proc. ... Annu. Int. Conf. IEEE Eng. Med. Biol. Soc. IEEE Eng. Med. Biol. Soc. Annu. Conf. 2015;2015:5428-31.

23. Ogisu K, Kudo K, Sasaki M, Sakushima K, Yabe I, Sasaki H, Terae S, Nakanishi M, Shirato H. 3D neuromelanin-sensitive magnetic resonance imaging with semi-automated volume measurement of the substantia nigra pars compacta for diagnosis of Parkinson's disease. Neuroradiology 2013;55:719-24.

24. Chen X, Huddleston DE, Langley J, Ahn S, Barnum CJ, Factor SA, Levey AI, Hu X. Simultaneous imaging of locus coeruleus and substantia nigra with a quantitative neuromelanin MRI approach. Magn. Reson. Imaging 2014;32:1301-6.

25. Henkelman RM, Huang X, Xiang QS, Stanisz GJ, Swanson SD, Bronskill MJ. Quantitative interpretation of magnetization transfer. Magn. Reson. Med. 1993;29:759-66.

26. van Buchem MA. Magnetization transfer: applications in neuroradiology. J. Comput. Assist. Tomogr. 1999;23 Suppl 1:S9-18.

27. Bryant R. The dynamics of water-protein interactions. Annu Rev Biophys Biomol Struct 1996;25:29-53.

28. Sled JG, Pike GB. Correction for B1 and B0 variations in quantitative T2 measurements using MRI. Magn. Reson. Med. 2000;43:589-593.

29. Sled JG, Pike GB. Quantitative imaging of magnetization transfer exchange and relaxation properties in vivo using MRI. Magn. Reson. Med. 2001;46:923-31.

30. Ramani A, Dalton C, Miller DH, Tofts PS, Barker GJ. Precise estimate of fundamental in-vivo MT parameters in human brain in clinically feasible times. Magn. Reson. Imaging 2002;20:721-731.

31. Yarnykh VL. Pulsed Z-spectroscopic imaging of cross-relaxation parameters in tissues for human MRI: theory and clinical applications. Magn. Reson. Med. 2002;47:929-39.

32. Yarnykh VL, Yuan C. Cross-relaxation imaging reveals detailed anatomy of white matter fiber tracts in the human brain. Neuroimage 2004;23:409-24.

33. Smith SA, Bulte JWM, van Zijl PCM. Direct saturation MRI: theory and application to imaging brain iron. Magn. Reson. Med. 2009;62:384-93.

34. Tosk JM, Holshouser BA, Aloia RC, Hinshaw DB, Hasso AN, MacMurray JP, Will AD, Bozzetti LP. Effects of the interaction between ferric iron and L-dopa melanin on T1 and T2 relaxation times determined by magnetic resonance imaging. Magn. Reson. Med. 1992;26:40-5. 
35. Enochs W, Petherick P, Bogdanova A, Mohr U, Weissleder R. Paramagnetic metal scavenging by melanin: MR imaging. Radiology 1997;204:417-23.

36. Enochs WS, Hyslop WB, Bennett HF, Brown RD, Koenig SH, Swartz HM. Sources of the increased longitudinal relaxation rates observed in melanotic melanoma. An in vitro study of synthetic melanins. Invest. Radiol. 1989;24:794-804.

37. Tanttu JI, Sepponen RE, Lipton MJ, Kuusela T. Synergistic enhancement of MRI with Gd-DTPA and magnetization transfer. J. Comput. Assist. Tomogr. 1992;16:19-24.

38. Helms G, Draganski B, Frackowiak R, Ashburner J, Weiskopf N. Improved segmentation of deep brain grey matter structures using magnetization transfer (MT) parameter maps. Neuroimage 2009;47:194-8.

39. Graham SJ, Henkelman RM. Understanding Pulsed Magnetization Transfer. J. Magn. Reson. Imaging 1997;7:903-912.

40. Morrison C, Henkelman R. A model for magnetization transfer in tissues. Magn Reson Med 1995;33:475482.

41. Smith SA, Farrell JAD, Jones CK, Reich DS, Calabresi PA, van Zijl PCM. Pulsed magnetization transfer imaging with body coil transmission at 3 Tesla: feasibility and application. Magn. Reson. Med. 2006;56:866-75.

42. Ferrari E, Engelen M, Monzani E, Sturini M, Girotto S, Bubacco L, Zecca L, Casella L. Synthesis and structural characterization of soluble neuromelanin analogs provides important clues to its biosynthesis. J. Biol. Inorg. Chem. 2013;18:81-93.

43. Laemmli UK. Cleavage of structural proteins during the assembly of the head of bacteriophage T4. Nature 1970;227:680-5.

44. Sled JG, Pike GB. Quantitative interpretation of magnetization transfer in spoiled gradient echo MRI sequences. J. Magn. Reson. 2000;145:24-36.

45. Skinner T, Glover G. An extended two-point Dixon algorithm for calculating separate water, fat, and B0 images. Magn Reson Med 1997;37:628-630.

46. Yarnykh V. Actual flip-angle imaging in the pulsed steady state: a method for rapid three-dimensional mapping of the transmitted radiofrequency field. Magn Reson Med 2007;57:192-200.

47. Fram EK, Herfkens RJ, Johnson GA, Glover GH, Karis JP, Shimakawa A, Perkins TG, Pelc NJ. Rapid calculation of T1 using variable flip angle gradient refocused imaging. Magn. Reson. Imaging 1987;5:201-208.

48. Kennan RP, Richardson KA, Zhong J, Maryanski MJ, Gore JC. The effects of cross-link density and chemical exchange on magnetization transfer in polyacrylamide gels. J. Magn. Reson. B 1996;110:267-77.

49. Aime S, Bergamasco B, Biglino D, Digilio G, Fasano M, Giamello E, Lopiano L. EPR investigations of the 
iron domain in neuromelanin. Biochim. Biophys. Acta 1997;1361:49-58.

50. Kropf AJ, Bunker BA, Eisner M, Moss SC, Zecca L, Stroppolo A, Crippa PR. X-ray absorption finestructure spectroscopy studies of Fe sites in natural human neuromelanin and synthetic analogues. Biophys. J. 1998;75:3135-42.

51. Zecca L, Swartz HM. Total and paramagnetic metals in human substantia nigra and its neuromelanin. J. Neural Transm. Park. Dis. Dement. Sect. 1993;5:203-13.

52. Gerlach M, Trautwein AX, Zecca L, Youdim MB, Riederer P. Mössbauer spectroscopic studies of purified human neuromelanin isolated from the substantia nigra. J. Neurochem. 1995;65:923-6.

53. Salustri C. Lack of Magnetization Transfer from the Ferritin Molecule. J. Magn. Reson. 1996;111:171-173.

54. Thomas SD, Al-Kwifi O, Emery DJ, Wilman AH. Application of magnetization transfer at $3.0 \mathrm{~T}$ in threedimensional time-of-flight magnetic resonance angiography of the intracranial arteries. J. Magn. Reson. Imaging 2002;15:479-83.

55. Morris CM, Edwardson JA. Iron histochemistry of the substantia nigra in Parkinson's disease. Neurodegeneration 1994;3:277-82.

56. Lehéricy S, Bardinet E, Poupon C, Vidailhet M, François C. 7 Tesla magnetic resonance imaging: a closer look at substantia nigra anatomy in Parkinson's disease. Mov. Disord. 2014;29:1574-1581.

57. Lewis MM, Du G, Kidacki M, Patel N, Shaffer ML, Mailman RB, Huang X. Higher iron in the red nucleus marks Parkinson's dyskinesia. Neurobiol Aging 2012;34:1497-503.

58. Zucca F, Bellei C, Giannelli S, Terreni M, Gallorini M, Rizzio E, Pezzoli G, Albertini A, Zecca L. Neuromelanin and iron in human locus coeruleus and substantia nigra during aging: consequences for neuronal vulnerability. J. Neural Transm. 2006;113:757-67.

59. Dexter DT, Wells FR, Agid F, Agid Y, Lees AJ, Jenner P, Marsden CD. Increased nigral iron content in postmortem parkinsonian brain. Lancet 1987;2:1219-20.

60. Dexter D, Wells F, Lees A, Agid F, Agid Y, Jenner P, Marsden C. Increased nigral iron content and alterations in other metal ions occurring in brain in Parkinson's disease. J. Neurochem. 1989;52:1830-6.

61. Earle K. Studies on Parkinson's disease including x-ray fluorescent spectroscopy of formalin fixed brain tissue. J. Neuropathol. Exp. Neurol. 1968;27:1-14.

62. Sofic E, Riederer P, Heinsen H, Beckmann H, Reynolds GP, Hebenstreit G, Youdim MB. Increased iron (III) and total iron content in post mortem substantia nigra of parkinsonian brain. J. Neural Transm. 1988;74:199205.

63. Jellinger K, Kienzl E, Rumpelmair G, Riederer P, Stachelberger H, Ben-Shachar D, Youdim M. Iron- 
melanin complex in substantia nigra of parkinsonian brains: an $\mathrm{x}$-ray microanalysis. J. Neurochem. 1992;59:1168-71.

64. Good P, Olanow C, Perl D. Neuromelanin-containing neurons of the substantia nigra accumulate iron and aluminum in Parkinson's disease: a LAMMA study. Brain Res. 1992;593:343-6.

65. Faucheux B, Martin M, Hauw J, Agid Y, Hirsch E, Beaumont C, Hauw J, Agid Y, Hirsch E. Neuromelanin associated redox-active iron is increased in the substantia nigra of patients with Parkinson's disease. J Neurochem 2003;86:1142-8.

66. Deistung A, Schäfer A, Schweser F, Biedermann U, Turner R, Reichenbach J. Toward in vivo histology: a comparison of quantitative susceptibility mapping (QSM) with magnitude-, phase-, and R2*-imaging at ultrahigh magnetic field strength. Neuroimage 2013;65:299-314.

67. Schweser F, Deistung A, Lehr B, Reichenbach J. Quantitative imaging of intrinsic magnetic tissue properties using MRI signal phase: an approach to in vivo brain iron metabolism? Neuroimage 2011;54:2789-807.

68. Zecca L, Costi P, Mecacci C, Ito S, Terreni M, Sonnino S. Interaction of human substantia nigra neuromelanin with lipids and peptides. J. Neurochem. 2000;74:1758-65.

69. Zucca FA, Segura-Aguilar J, Ferrari E, Muñoz P, Paris I, Sulzer D, Sarna T, Casella L, Zecca L. Interactions of iron, dopamine and neuromelanin pathways in brain aging and parkinson's disease. Prog. Neurobiol. 2015. 


\section{Figure Legends}

Figure 1. Simulated MTR-spectra with variable parameters. (a) $T_{1}^{f}=0.25,0.5,0.75,1 \mathrm{~s}$; (b) same as (a) without MT effect (only DS effect); (c) $T_{2}^{f}=30,60,120,240 \mathrm{~ms}$; (d) same as (c) without MT effect; (e) $T_{2}^{m}=8$, $14,20,26 \mu \mathrm{s}$; (f) $M_{0}^{m}=0.5 \%, 1 \%, 3 \%, 6 \%$; (g) $k_{f m}=0.1,0.5,1,10 \mathrm{~s}^{-1}$; (h) $\alpha_{\mathrm{MT}}=400^{\circ}, 650^{\circ}, 900^{\circ}, 1150^{\circ}$. The rest of the parameters for all plots were fixed, corresponding to the MT experimental protocol (25-ms sinc-gauss,

$\alpha_{\mathrm{MT}}=1150^{\circ}$, excitation flip angle $=10^{\circ}, \mathrm{TR}=58 \mathrm{~ms}$ ) and the following set of spin-system parameters: $T_{1}^{f}=1 \mathrm{~s}$, $T_{2}^{f}=240 \mathrm{~ms}, T_{2}^{m}=14 \mu \mathrm{s}, M_{0}^{m}=1 \%, k_{f m}=1 \mathrm{~s}^{-1}$. For (b) and (d), $k_{f m}=0 \mathrm{~s}^{-1}$. In all cases, the absorption lineshape for the macromolecular pool was chosen to be Super-Lorentzian. In all plots, the black line shows the MTR-spectra using the default parameters.

Figure 2. Relaxation rates of samples in polyacrylamide containing different concentrations of melanin and iron. (a) Longitudinal relation rate $\left(R_{1}^{o b s}=1 / T_{1}^{o b s}\right)$; (b) Transverse relaxation rate $\left(R_{2}^{o b s}=1 / T_{2}^{o b s}\right)$.

Figure 3. Relaxation rates of samples in polyacrylamide plotted against iron concentration. (a) Longitudinal relation rate $\left(R_{1}^{o b s}=1 / T_{1}^{o b s}\right) ;(\mathrm{b})$ Transverse relaxation rate $\left(R_{2}^{o b s}=1 / T_{2}^{o b s}\right)$.

Figure 4. Experimental MTR-spectra for selected samples in polyacrylamide. (a) metal-free melanin at different concentrations; (b) different metallomelanins at the same concentration $(3.5 \mathrm{mg} / \mathrm{ml})$; (c) metallomelanin 4 at different concentrations. Error bars represent one standard deviation. In all cases, the offset frequency was corrected using the $\mathrm{B}_{0}$ map.

Figure 5. Experimental and simulated MTR-spectra for the samples prepared in 4\% agarose. (a) Experimental spectra with corrected offset frequency (error bars represent one standard deviation); (b) simulated MTR-spectra using the same parameters as the MT experimental protocol (see methods), fixed $T_{2}^{m}=14 \mu \mathrm{s}, k_{f m}=1 \mathrm{~s}-1, M_{0}^{f}=$ $1, M_{0}^{m}=0.015$, and the measured $T_{1}^{o b s}$ and $T_{2}^{o b s}$ of the corresponding sample. The concentration for all the melanins showed in the figure was $3.5 \mathrm{mg} / \mathrm{ml}$.

Figure 6. (a) MTR calculated for the MT data obtained with $\alpha_{\mathrm{MT}}=1150^{\circ}$, and $\Delta \omega=1.5 \mathrm{kHz}$; (b) CNR calculated from the NM-MRI images. 
Supporting Figure S1. Prepared phantom. (a) Prepared samples, including (left to right): 11 samples with the reference gel, 4 samples with only iron, 4 samples of metal-free melanin, 16 samples with metallomelanins, and 2 samples with ultra-pure water. (b)-(c) Samples positioned in a hexagonal grid. (d) Samples immersed in a bath of gadolinium doped gelatin.

Supporting Figure S2. Results from the direct saturation fitting. (a) Linewidth; (b) DSR calculated at $\Delta \omega=250$ $\mathrm{Hz}$. 


\section{Tables}

\begin{tabular}{llll} 
Sample & $\begin{array}{l}\text { L-Cys/DA } \\
(\text { molar ratio })\end{array}$ & $\begin{array}{l}\text { Initial iron/DA (molar } \\
\text { ratio) }\end{array}$ & Total iron measured by ICP-OES $(\mu \mathrm{g} / \mathrm{mg})$ \\
\hline Metal-free melanin & 0.2 & - & Below limit of detectability \\
Metallomelanin 1 & 0.2 & 0.025 & $9.98 \pm 0.01$ \\
Metallomelanin 2 & 0.2 & 0.05 & $11.4 \pm 0.7$ \\
Metallomelanin 3 & 0.2 & 0.075 & $19.1 \pm 0.9$ \\
Metallomelanin 4 & 0.2 & 0.1 & $23.3 \pm 1.4$
\end{tabular}

Table 1. Synthesis conditions of metal-free melanin and metallomelanins used in this study. The second column shows the molar ratio of dopamine and L-Cysteine used to synthesize melanins. The initial molar ratio between iron and dopamine is reported in the third column, while the iron load in synthesized melanins is shown in the last column. 


\begin{tabular}{|c|c|c|c|c|c|c|c|}
\hline \multirow{2}{*}{ Description } & \multirow{2}{*}{$\begin{array}{l}\text { Melanin } \\
(\mathrm{mg} / \mathrm{ml})\end{array}$} & \multirow{2}{*}{$\underset{(\mu \mathrm{g} / \mathrm{ml})}{\text { Iron }}$} & \multicolumn{2}{|c|}{ Relaxometry } & \multicolumn{3}{|c|}{ qMT } \\
\hline & & & $T_{1}^{o b s}(\mathrm{~ms})$ & $T_{2}^{o b s}(\mathrm{~ms})$ & $\operatorname{PSR}(\%)$ & $T_{2}^{f}(m s)$ & $\mathbf{T}_{2}^{\mathrm{m}}(\boldsymbol{\mu s})$ \\
\hline Reference* & 0 & 0 & $1042 \pm 23$ & $258.69 \pm 3.41$ & $0.76 \pm 0.09$ & $90.11 \pm 33.33$ & $46.27 \pm 16.22$ \\
\hline \multirow{4}{*}{ Iron } & 0 & $34.93 \pm 0.01$ & $648 \pm 12$ & $219.65 \pm 1.89$ & $0.85 \pm 0.13$ & $63.08 \pm 2.38$ & $12.93 \pm 0.51$ \\
\hline & 0 & $39.91 \pm 0.01$ & $591 \pm 9$ & $204.72 \pm 0.91$ & $0.77 \pm 0.15$ & $52.53 \pm 23.45$ & $12.10 \pm 1.24$ \\
\hline & 0 & $66.77 \pm 0.01$ & $420 \pm 5$ & $165.77 \pm 0.49$ & $0.86 \pm 0.16$ & $48.23 \pm 8.99$ & $13.27 \pm 4.75$ \\
\hline & 0 & $81.66 \pm 0.01$ & $344 \pm 2$ & $149.22 \pm 0.51$ & $0.83 \pm 0.27$ & $51.38 \pm 6.29$ & $11.15 \pm 0.47$ \\
\hline \multirow{4}{*}{ Metal-free Melanin } & $0.88 \pm 0.01$ & 0 & $1037 \pm 27$ & $240.43 \pm 2.08$ & $0.72 \pm 0.05$ & $88.45 \pm 22.88$ & $34.00 \pm 14.81$ \\
\hline & $1.75 \pm 0.01$ & 0 & $1105 \pm 48$ & $227.17 \pm 2.79$ & $0.59 \pm 0.11$ & $64.00 \pm 15.19$ & $31.83 \pm 26.46$ \\
\hline & $2.63 \pm 0.02$ & 0 & $1036 \pm 24$ & $215.41 \pm 2.47$ & $0.72 \pm 0.13$ & $52.45 \pm 5.49$ & $43.55 \pm 15.39$ \\
\hline & $3.50 \pm 0.02$ & 0 & $1186 \pm 32$ & $210.66 \pm 1.54$ & $0.52 \pm 0.07$ & $74.76 \pm 14.46$ & $31.23 \pm 22.90$ \\
\hline \multirow{4}{*}{ Metallomelanin 1} & $0.88 \pm 0.01$ & $8.73 \pm 0.01$ & $704 \pm 16$ & $112.08 \pm 0.60$ & $0.77 \pm 0.22$ & $46.84 \pm 4.34$ & $23.73 \pm 4.39$ \\
\hline & $1.75 \pm 0.01$ & $17.46 \pm 0.02$ & $535 \pm 13$ & $78.06 \pm 0.58$ & $0.74 \pm 0.24$ & $44.32 \pm 2.21$ & $26.80 \pm 7.76$ \\
\hline & $2.63 \pm 0.02$ & $26.19 \pm 0.03$ & $426 \pm 5$ & $57.54 \pm 0.52$ & $1.13 \pm 0.65$ & $35.95 \pm 1.94$ & $26.69 \pm 1.14$ \\
\hline & $3.50 \pm 0.02$ & $34.93 \pm 0.04$ & $374 \pm 7$ & $46.54 \pm 0.39$ & $1.10 \pm 0.40$ & $34.89 \pm 2.66$ & $5.48 \pm 2.11$ \\
\hline \multirow{4}{*}{ Metallomelanin 2} & $0.88 \pm 0.01$ & $9.98 \pm 0.59$ & $608 \pm 10$ & $109.83 \pm 0.89$ & $0.92 \pm 0.38$ & $47.36 \pm 2.22$ & $51.92 \pm 1.08$ \\
\hline & $1.75 \pm 0.01$ & $19.96 \pm 1.16$ & $376 \pm 9$ & $70.05 \pm 0.45$ & $0.90 \pm 0.14$ & $46.35 \pm 6.10$ & $45.97 \pm 16.73$ \\
\hline & $2.63 \pm 0.02$ & $29.93 \pm 1.73$ & $321 \pm 4$ & $54.79 \pm 0.93$ & $1.15 \pm 0.39$ & $41.12 \pm 3.96$ & $23.45 \pm 1.48$ \\
\hline & $3.50 \pm 0.02$ & $39.91 \pm 2.31$ & $256 \pm 5$ & $43.53 \pm 0.70$ & $1.49 \pm 0.31$ & $41.96 \pm 4.97$ & $13.75 \pm 0.51$ \\
\hline \multirow{4}{*}{ Metallomelanin 3} & $0.88 \pm 0.01$ & $16.69 \pm 0.82$ & $533 \pm 9$ & $100.07 \pm 1.19$ & $0.95 \pm 0.21$ & $48.64 \pm 7.08$ & $32.43 \pm 6.54$ \\
\hline & $1.75 \pm 0.01$ & $33.39 \pm 1.63$ & $323 \pm 13$ & $62.05 \pm 0.60$ & $0.87 \pm 0.19$ & $39.90 \pm 2.46$ & $30.90 \pm 1.66$ \\
\hline & $2.63 \pm 0.02$ & $50.08 \pm 2.45$ & $267 \pm 3$ & $44.03 \pm 0.41$ & $0.80 \pm 0.20$ & $45.55 \pm 2.70$ & $33.23 \pm 3.50$ \\
\hline & $3.50 \pm 0.02$ & $66.77 \pm 3.26$ & $194 \pm 4$ & $35.03 \pm 0.62$ & $0.60 \pm 0.36$ & $40.49 \pm 4.92$ & $4.49 \pm 0.49$ \\
\hline \multirow{4}{*}{ Metallomelanin 4} & $0.88 \pm 0.01$ & $20.42 \pm 1.19$ & $417 \pm 7$ & $84.06 \pm 0.65$ & $0.97 \pm 0.49$ & $43.37 \pm 3.80$ & $33.64 \pm 1.75$ \\
\hline & $1.75 \pm 0.01$ & $40.83 \pm 2.39$ & $286 \pm 5$ & $53.04 \pm 0.68$ & $0.99 \pm 0.32$ & $49.87 \pm 5.26$ & $15.96 \pm 1.56$ \\
\hline & $2.63 \pm 0.02$ & $61.25 \pm 3.58$ & $199 \pm 3$ & $37.53 \pm 0.56$ & $1.34 \pm 0.60$ & $43.11 \pm 6.69$ & $15.81 \pm 1.64$ \\
\hline & $3.50 \pm 0.02$ & $81.66 \pm 4.77$ & $161 \pm 3$ & $29.02 \pm 0.50$ & $1.00 \pm 0.20$ & $38.30 \pm 8.26$ & $4.27 \pm 0.54$ \\
\hline
\end{tabular}

Table 2. Samples prepared in polyacrylamide. The second and third columns indicate the concentration of melanin and iron, respectively. The fourth and fifth columns show the measured $T_{1}^{o b s}$ and $T_{2}^{o b s}$, and the last three columns show the results from the qMT fitting. Data are mean \pm standard deviation

* $10 \%$ BSA in $10 \%$ polyacrylamide 


\begin{tabular}{lccccccc}
\multirow{2}{*}{ Description } & \multirow{2}{*}{$\begin{array}{c}\text { Melanin } \\
(\mathbf{m g} / \mathbf{m l})\end{array}$} & $\begin{array}{c}\text { Iron } \\
(\boldsymbol{\mu g} / \mathbf{m l})\end{array}$ & $\boldsymbol{T}_{\mathbf{1}}^{\boldsymbol{o b s}}(\mathbf{m s})$ & $\boldsymbol{T}_{\mathbf{2}}^{\boldsymbol{o b s}}(\mathbf{m s})$ & $\mathbf{P S R}(\boldsymbol{\%})$ & $\mathbf{T}_{\mathbf{2}}^{\mathbf{f}}(\mathbf{m s})$ & $\mathbf{T}_{\mathbf{2}}^{\mathbf{m}}(\boldsymbol{\mu s})$ \\
\hline 4\% agarose & 0 & 0 & $1888 \pm 121$ & $35.47 \pm 0.68$ & $1.43 \pm 0.16$ & $39.60 \pm 2.65$ & $15.24 \pm 2.53$ \\
\hline \multirow{2}{*}{ Iron } & 0 & $34.93 \pm 0.02$ & $547 \pm 10$ & $32.09 \pm 0.36$ & $0.98 \pm 0.20$ & $44.91 \pm 1.93$ & $18.16 \pm 3.23$ \\
& 0 & $39.91 \pm 0.02$ & $525 \pm 5$ & $32.08 \pm 0.43$ & $1.14 \pm 0.22$ & $44.88 \pm 1.46$ & $18.86 \pm 2.82$ \\
& 0 & $66.77 \pm 0.02$ & $358 \pm 6$ & $28.37 \pm 0.37$ & $0.95 \pm 0.32$ & $42.07 \pm 1.97$ & $17.67 \pm 3.50$ \\
& 0 & $81.66 \pm 0.02$ & $305 \pm 3$ & $28.65 \pm 0.39$ & $0.90 \pm 0.22$ & $38.38 \pm 2.64$ & $17.45 \pm 4.59$ \\
\hline \multirow{2}{*}{ Metal-free melanin } & $1.75 \pm 0.02$ & 0 & $1906 \pm 264$ & $33.88 \pm 0.90$ & $1.13 \pm 0.06$ & $42.02 \pm 3.25$ & $14.83 \pm 1.18$ \\
& $3.50 \pm 0.04$ & 0 & $1818 \pm 38$ & $32.07 \pm 0.58$ & $1.17 \pm 0.19$ & $38.54 \pm 2.11$ & $17.32 \pm 1.90$ \\
\hline Metallomelanin 1 & $3.50 \pm 0.04$ & $34.93 \pm 0.04$ & $512 \pm 16$ & $16.35 \pm 0.72$ & $1.26 \pm 0.21$ & $32.62 \pm 2.31$ & $8.79 \pm 2.24$ \\
\hline Metallomelanin 2 & $3.50 \pm 0.04$ & $39.91 \pm 2.31$ & $274 \pm 8$ & $18.76 \pm 0.40$ & $1.14 \pm 0.49$ & $26.90 \pm 3.68$ & $7.02 \pm 3.65$ \\
\hline \multirow{2}{*}{ Metallomelanin 3 } & $1.75 \pm 0.02$ & $33.39 \pm 1.63$ & $308 \pm 6$ & $19.34 \pm 0.18$ & $1.16 \pm 0.59$ & $29.01 \pm 3.04$ & $9.01 \pm 4.31$ \\
& $3.50 \pm 0.04$ & $66.77 \pm 3.26$ & $194 \pm 6$ & $14.54 \pm 0.86$ & $1.12 \pm 0.32$ & $19.98 \pm 3.10$ & $4.07 \pm 3.74$ \\
\hline \multirow{2}{*}{ Metallomelanin 4 } & $1.75 \pm 0.02$ & $40.83 \pm 2.39$ & $288 \pm 7$ & $18.52 \pm 0.55$ & $1.67 \pm 0.53$ & $25.03 \pm 2.01$ & $8.57 \pm 3.66$ \\
& $3.50 \pm 0.04$ & $81.66 \pm 4.77$ & $184 \pm 5$ & $13.55 \pm 0.60$ & $0.98 \pm 0.43$ & $16.40 \pm 3.10$ & $5.83 \pm 2.37$ \\
\hline
\end{tabular}

Table 3. Samples prepared in $4 \%$ agarose. The second and third columns indicate the concentrations of melanin and iron, respectively. The fourth and fifth columns show the measured $T_{1}^{o b s}$ and $T_{2}^{o b s}$, and the last three columns show the results from the qMT fitting. Data are mean \pm standard deviation. 